Article

\title{
A Patient-Specific Study Investigating the Relation between Coronary Hemodynamics and Neo-Intimal Thickening after Bifurcation Stenting with a Polymeric Bioresorbable Scaffold
}

\author{
Susanna Migliori ${ }^{1,+}+\left(\right.$ i) , Rajiv Rampat ${ }^{2,+}$, Marco Bologna ${ }^{1,3}$, Eros Montin ${ }^{3,4}$, \\ Francesco Burzotta ${ }^{5}$, David Hildick-Smith ${ }^{2}$, Gabriele Dubini ${ }^{1}$, Luca Mainardi ${ }^{3}$ (i), \\ Francesco Migliavacca ${ }^{1}$, James Cockburn ${ }^{2}$ and Claudio Chiastra ${ }^{1, *}$ (iD) \\ 1 Laboratory of Biological Structure Mechanics (LaBS), Department of Chemistry, Materials and Chemical \\ Engineering “Giulio Natta”, Politecnico di Milano, 20133 Milan, Italy; susanna.migliori@polimi.it (S.M.); \\ marco.bologna@polimi.it (M.B.); gabriele.dubini@polimi.it (G.D.); francesco.migliavacca@polimi.it (F.M.) \\ 2 Sussex Cardiac Centre, Brighton and Sussex University Hospitals, Brighton BN2 5BE, UK; \\ rampat@doctors.org.uk (R.R.); david.hildick-smith@bsuh.nhs.uk (D.H.-S.); \\ james.cockburn@bsuh.nhs.uk (J.C.) \\ 3 Department of Electronics, Information and Bioengineering, Politecnico di Milano, 20133 Milan, Italy; \\ eros.montin@gmail.com (E.M.); luca.mainardi@polimi.it (L.M.) \\ 4 Center for Advanced Imaging Innovation and Research (CAI2R), and the Bernard and Irene Schwartz \\ Center for Biomedical Imaging, Department of Radiology, New York University School of Medicine, \\ New York, NY 10016, USA \\ 5 Institute of Cardiology, Fondazione Policlinico Universitario A. Gemelli IRCCS, Università Cattolica del \\ Sacro Cuore, 00168 Rome, Italy; francesco.burzotta@unicatt.it \\ * Correspondence: claudio.chiastra@polimi.it; Tel.: +39-02-2399-3169 \\ + First authorship shared.
}

Received: 17 July 2018; Accepted: 29 August 2018; Published: 1 September 2018

\begin{abstract}
We present an application of a validated reconstruction methodology for the comparison between patient-specific hemodynamics and neo-intimal thickening at nine months from the intervention. (1) Background: Coronary bifurcation stenting alters the vessel geometry, influencing the local hemodynamics. The evaluation of wall shear stress (WSS) relies on the application of computational fluid dynamics to model its distribution along the coronary tree. The endothelium actively responds to WSS, which triggers eventual cell proliferation to cover the stent struts. (2) Methods: Baseline optical coherence tomography and angiographic data were combined to reconstruct a patient-specific coronary bifurcation with an implanted bioresorbable scaffold and to simulate the hemodynamics. Results were linked with the neo-intimal thickening after nine months from the intervention. (3) Results: Blood velocity patterns were disrupted at the bifurcation due to the presence of the stent. It was observed that $55.6 \%$ of the scaffolded lumen surface was exposed to values of time-averaged WSS lower than 0.4 Pa. Follow-up images showed a luminal narrowing of $19 \%$ in the main branch. There was also a complete coverage in $99 \%$ of struts. (4) Conclusions: This approach provided valuable complementary information that might improve the clinical outcomes in this subset of coronary diseases.
\end{abstract}

Keywords: coronary artery bifurcation; stent; bioresorbable scaffold; optical coherence tomography; image segmentation; three-dimensional reconstruction; patient-specific computer modeling; computational fluid dynamics; neo-intimal coverage; wall shear stress 


\section{Introduction}

A coronary stent is a wire mesh or cut tubular structure inserted into a stenosed or blocked coronary artery to improve blood flow to the myocardium subtended by that artery. In the past years, bioresorbable coronary devices have been developed, seeking to overcome the limitations of the state-of-the-art drug-eluting permanent stents, including the risks of target lesion revascularization, neo-atherosclerosis, hindrance of late lumen enlargement, and the lack of reactive vasomotion in the stented vessel [1]. These devices, which are referred to as bioresorbable scaffolds, fully dissolve in the vessel wall in two or three years after recovering the lumen passageway [1].

The ideal bioresorbable scaffold re-opens the artery without any long-term adverse sequelae, such as restenosis or thrombosis. Vascular tissue re-modelling after stent implantation is influenced by the local blood flow pattern [2]. The endothelium actively responds to wall shear stress (WSS), which triggers vascular smooth muscle cell proliferation and migration to the endoluminal surface, causing vessel healing and strut coverage. One method to quantify strut coverage is by measuring the neo-intimal thickening (NIT), defined as the perpendicular distance between the midpoint of the adluminal strut surface and the inner luminal surface measured on optical coherence tomography (OCT) images [2]. Another method is to calculate the restenosis area, defined as the difference between the scaffold area and the sum of the strut and luminal area at a particular cross-section of the OCT on follow-up imaging. The high in-plane resolution of OCT (axial resolution between 12 and $15 \mu \mathrm{m}$, and lateral resolution between 20 and $40 \mu \mathrm{m}$ [3]) allows a detailed visualization of the stent struts and evaluation of the neointimal layer at follow-up [4]. The evaluation of WSS relies on the application of computational fluid dynamics (CFD) to model patient's hemodynamics. The incidence of adverse neointimal hyperplastic response may be predicted using surrogate quantities, such as a low WSS averaged over one cardiac cycle $(<0.4 \mathrm{~Pa})[5]$ and disrupted flow stream at bifurcation.

In the present study, we sought to investigate the relation between patient's hemodynamics at baseline and NIT after nine-month from the intervention. A previously validated optical coherence tomography (OCT) based methodology [6] was applied to reconstruct a patient-specific coronary artery at baseline, after the insertion of a bioresorbable polymeric scaffold. The achieved high fidelity geometrical model was used to simulate the blood flow under the effect of the scaffold. The lumen contour was traced in the OCT image datasets at baseline and follow-up to compute the relative distance. Results of the CFD simulation were linked to the neo-intimal coverage, using the NIT and restenosis area.

\section{Materials and Methods}

\subsection{Image Data Collection}

A 57 year old lady presenting with stable angina was found to have an occlusive disease at the bifurcation between the left anterior descending (LAD) and the first diagonal coronary artery (Medina ' $0,1,0^{\prime}$ type [7]). Predilatation of the lesion was performed with a $2.5 \times 12 \mathrm{~mm}$ angioplasty balloon. OCT imaging was performed after balloon dilatation using the FastView ${ }^{\circledR}$ catheter and the Lunawave ${ }^{\circledR}$ coronary imaging console (Terumo Corp., Tokyo, Japan). Contrast was injected at a rate of $4 \mathrm{~mL} / \mathrm{s}$ and OCT images were acquired at a rate of 158 frames s${ }^{-1}$ with a pullback speed of $20 \mathrm{~mm} \mathrm{~s}^{-1}$. Proximal and distal reference diameters were $2.90 \mathrm{~mm}$ and $2.22 \mathrm{~mm}$, respectively. A $3.0 \times 18 \mathrm{~mm}$ Absorb Bioresorbable Vascular Scaffold (BVS) (Abbott Vascular, Abbott Park, IL, USA) was implanted in the main branch at $12 \mathrm{~atm}$. This bioresorbable scaffold is made of poly-L-lactic acid and it is coated with a poly-D,L-lactide layer that regulates the release of the anti-proliferative drug everolimus [1]. The scaffold struts are rectangular with a thickness of $\sim 157 \mu \mathrm{m}$, which ensures good radial strength for a polymeric device [1]. Proximal optimization technique was performed with a $3.25 \times 8 \mathrm{~mm}$ non-compliant balloon inflated to $14 \mathrm{~atm}$. Post implant OCT showed good scaffold apposition across the main branch with no edge dissection. Final quantitative coronary angiography (QCA) analysis 
showed a residual $12 \%$ stenosis at the side branch ostium and $28 \%$ within the LAD. OCT imaging was repeated at 9 months after scaffold implantation.

\subsection{Reconstruction of the Stent Coronary Artery Bifurcation}

\subsubsection{Image Segmentation}

The three-dimensional (3D) geometry of the patient's bifurcation with the implanted Absorb BVS was reconstructed by means of a validated OCT-based methodology [6]. In particular, an automatic segmentation algorithm was implemented to detect the baseline lumen contour and the polymeric scaffold in the baseline OCT image set (Figure 1A). The algorithm comprised four main steps: (i) Image pre-preprocessing; (ii) lumen segmentation; (iii) scaffold struts segmentation; and (iv) strut-based lumen correction.

Image pre-processing followed the strategy that was introduced by Wang and colleagues [8]. Briefly, the OCT catheter and the noise inside the lumen were removed. This was achieved with a binary mask that was created through the following steps: Intensity thresholding, voxels with intensity below a threshold were neglected; morphological opening, uniform clusters of high intensity pixels were created; and area thresholding, isolated small bright regions were neglected.

The lumen contours were segmented according to a previously described strategy $[6,9]$. The pre-processed images were elaborated in polar coordinates and a Sobel filter was applied to detect edges. The first pixel detected as an edge for each image column (i.e., for each A-scan) was considered as part of the lumen contour. The identified pixels were interpolated and were converted back to Cartesian coordinates (Figure 1B).

The scaffold struts segmentation was entirely performed in Cartesian coordinates. The criterion for scaffold identification relied on the fact that polymeric stent struts appear in the OCT images as approximately rectangular regions with a low intensity core surrounded by bright pixels. Hence, the struts were identified with three steps: (i) Intensity thresholding, by using a pre-defined quantile of the pixel intensity distribution; (ii) area filling operation, to close the holes in the binary image; (iii) Boolean subtraction of the original binary image from the filled image. When the scaffold struts presented a partially connected layer of bright pixels at their boundary (i.e., "open box" struts), the procedure described above failed to recognize the struts. Furthermore, false positives were identified with the implemented segmentation strategy, especially close to the outer wall.

The problem introduced by "open-box" struts was addressed by an intensity thresholding step followed by a morphological closing with a disk structure element of 1-pixel radius (1 pixel is equal to $\sim 15 \mu \mathrm{m}$ ). Furthermore, a nonlinear filter ( $\gamma$-filter, with $\gamma=0.6$ ) was applied to the images to enhance the image contrast. The correction of detected stent false positives comprised a series of filtering operations. Firstly, the area of acceptable struts candidates was bounded between two thresholds (e.g., 10 and 300 pixels). Secondly, the lumen was dilated using a disk of radius 20 pixels as tolerance for struts position; therefore, all the struts outside such a region were removed. Lastly, centroids of the remaining strut candidates were interpolated with a spline and stent false positives at a distance lager than 5 pixels were neglected. The resultant region of interest was composed of the actual stent struts (i.e., stent true positives) (Figure 1C).

The strut-based lumen correction was the last step of the segmentation process. The lumen contour at baseline was obtained by combining the lumen boundary previously identified with the line that interpolates the position of the abluminal wall of the struts. This analysis was performed in polar coordinates. For each A-scan image (i.e., one-dimensional depth profile), only the contour with the highest radial value was chosen (Figure 1D).

\subsubsection{Geometry Reconstruction}

The segmentation of the OCT images provided the point clouds for the main branch lumen and the implanted stent that were placed along the 3D vessel centerline to build the 3D stented 
coronary bifurcation geometry. The vessel centerline was extracted from angiographic images that were elaborated with the commercial software CAAS (Pie Medical Imaging BV, Maastricht, The Netherlands). Then, the 3D geometries of the vessel and the scaffold struts were obtained by following a previously developed and validated 3D reconstruction methodology [6].
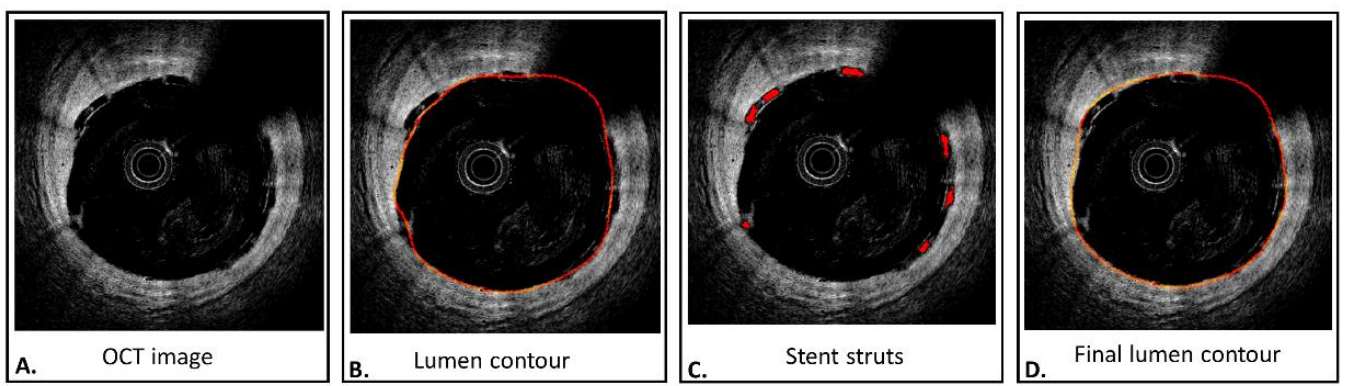

Figure 1. Main steps of the segmentation algorithm for the detection of lumen contours and polymeric stent struts. (A): Optical coherence tomography (OCT) frame of a coronary artery treated with the Absorb BVS (Abbott Vascular). (B): Detected lumen contour (a dilatation of 1 pixel has been applied to enable the edge visualization). (C): Regions of interests for the detected scaffold struts. (D): Final lumen contour after the correction with the scaffold struts (a dilatation of 1 pixel has been applied to enable edge visualization).

The surface for the vessel main branch was defined as follows. The lumen contour curves were built from a point interpolation and, then, were used to create the lumen surface with a lofting procedure. The side branches were reconstructed based on information achieved with a 3D-QCA that was carried out with the same software used for the elaboration of the angiographic images. Essentially, the side branch cross-section was created by scaling the main branch cross-section area to preserve the ratio between main branch and side branch cross-section areas of the 3D-QCA geometry. The length of each side branch was 2.5 times the side branch cross-section diameter.

The Absorb BVS skeleton (i.e., the stent wire) was drawn in the CAD software Rhinoceros v.5 (Robert McNeel \& Associates, Seattle, WA, USA) as a sequence of sinusoidal rings connected with straight bridges, in order to resemble the actual Absorb BVS design. High resolution pictures were taken during an in-vitro free expansion of an Absorb BVS. These pictures were used to extract useful measurements for the customization of the sinusoidal function, so that the stent crowns were correctly resembled. The final 3D reconstruction of the implanted scaffold was achieved by means of the graphical algorithm editor Grasshopper 3D ${ }^{\circledR}$ within Rhinoceros. In particular, the cross-section curves were rectangles with sides of $0.905 \times 0.1575 \mathrm{~mm}$ for the rings and $0.1397 \times 0.1575 \mathrm{~mm}$ for the bridges.

The final geometry, which resembled scaffolded patient's coronary bifurcation, comprised a $3.0 \times 18 \mathrm{~mm}$ Absorb BVS and a $44.3 \mathrm{~mm}$ main branch with two side branches (Figure 2).

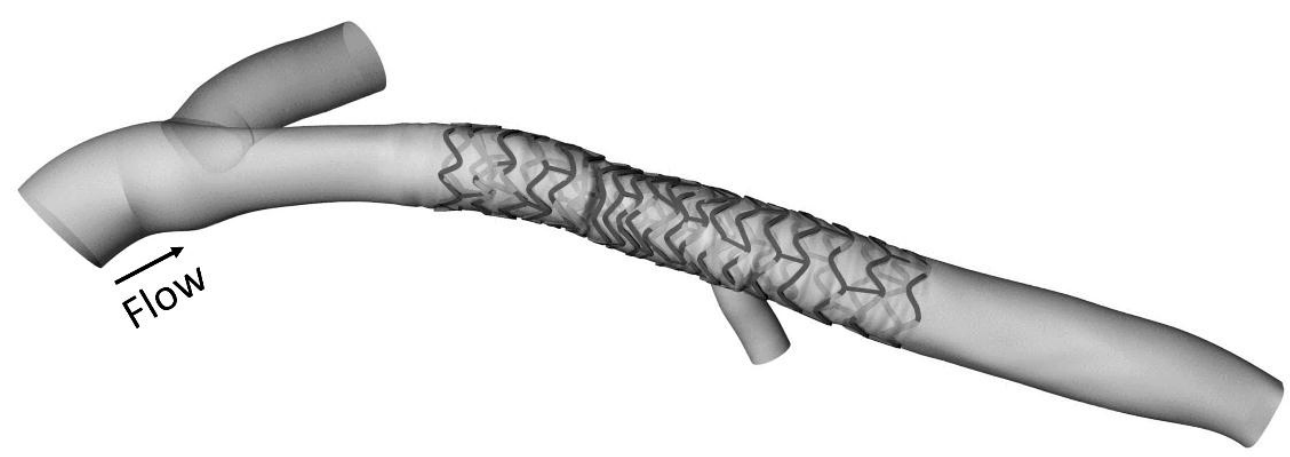

Figure 2. Reconstruction of the scaffolded coronary artery segment with the direction of the simulated blood flow. 


\subsection{Computational Fluid Dynamics Model}

The physical domain was discretized in $3.305 \times 10^{6}$ tetrahedral elements using ICEM CFD v.18 (ANSYS Inc., Canonsburg, PA, USA). The smallest element size was chosen according to previous mesh independence studies on stented coronary artery models [10,11] and was approximately one seventh of the strut thickness, which enabled a good description of the blood flow at the scaffolded segment. The hemodynamic field was computed by solving the governing equations of the unsteady, incompressible fluid motion with the commercial finite volume based software Fluent v.18 (ANSYS Inc.). A typical human LAD flow waveform [12] (mean flow-rate of $90.17 \mathrm{~mL} \mathrm{~min}^{-1}$ ) was applied at the inlet. The mean flow-rate was estimated from patient's angiography using a frame count method, which accounts for the number of cine frames with the radio-opaque liquid flowing in a coronary artery segment [13]. A flow-split 44.2\%:9.5\%:6.4\% for the distal main branch and side branches, respectively, was imposed at the outlets [14]. No slip-condition was applied to the arterial and scaffold walls, which were defined as rigid. The blood was described as an incompressible, non-Newtonian fluid, implementing a Bird-Carreau constitutive law with $\mu_{\infty}=0.0035 \mathrm{~kg} \mathrm{~m}^{-1} \mathrm{~s}^{-1}, \mu_{0}=0.25 \mathrm{~kg} \mathrm{~m}^{-1} \mathrm{~s}^{-1}$, $\lambda=25 \mathrm{~s}, \mathrm{n}=0.25$, and a density of $1060 \mathrm{~kg} \mathrm{~m}^{-3}$ [15]. The solver settings are reported in [10].

The local hemodynamics of the patient-specific case was analyzed in terms of WSS descriptors. In particular, given the definition of WSS:

$$
\vec{\tau}_{w}=\vec{n} \cdot \overrightarrow{\tau_{i j}}
$$

where $\vec{n}$ is the normal vector to the arterial wall surface and $\overrightarrow{\tau_{i j}}$ is the fluid viscous stress tensor, the time-averaged WSS (TAWSS) was calculated as [16]:

$$
\text { TAWSS }=\frac{1}{T} \int_{0}^{T}\left|\vec{\tau}_{w}\right| d t
$$

where $T$ is the duration of cardiac cycle. Therefore, the TAWSS enables to analyze the overall effects of the temporal variation of the WSS over the cardiac cycle along the coronary artery lumen.

\subsection{Quantification of Neointimal Thickness}

OCT analyses were performed offline with a software provided by Terumo (Terumo Corp.) and an in-house automatic segmentation algorithm. The commercial software was employed to compute the distance between baseline and follow-up lumen contours that were obtained from the corresponding OCT acquisitions (i.e., during the intervention and after 9 months). In particular, the OCT frames were acquired at $0.125 \mathrm{~mm}$ intervals (i.e., 8 frames per $\mathrm{mm}$ ). The stented segments, including edges of $5 \mathrm{~mm}$ on either side of the scaffold, were analyzed at $1 \mathrm{~mm}$ intervals (i.e., 1 frame out of 8 frames). Moreover, the OCT image dataset at 9-months follow-up was elaborated in the MATLAB environment (Mathworks, Natick, MA, USA) to compute the scaffold coverage area. Briefly, the follow-up lumen contour in each OCT frame was the innermost lumen boundary (Figure 3A); the stent struts were used as guidance for the identification of the baseline lumen contour (Figure 3B). In some cases, malapposed scaffold struts at baseline appeared completely covered by neointima in the follow-up acquisition. For such cases, the baseline lumen contours for the computation of the NIT were traced as shown in Figure 3C, as the high image resolution enabled to distinguish the grown layer of vascular smooth muscle cells. Whereas, the malapposed stent struts at follow-up were not considered for the computation of the NIT. A similar approach was used by Bourantas and colleagues [17] to compute the neo-intima thickening after 1-year follow-up. Thus, the regions that were assumed as NIT are reported in Figure 3D. The tissue re-modelling was quantified by the ratio between NIT area and the baseline lumen area, reported as the percentage of restenosis area. Such evaluation was performed at 
the scaffolded portion of the main branch, which was divided in proximal and distal regions, with respect to the side branch, and in bifurcation region, at the side branch.

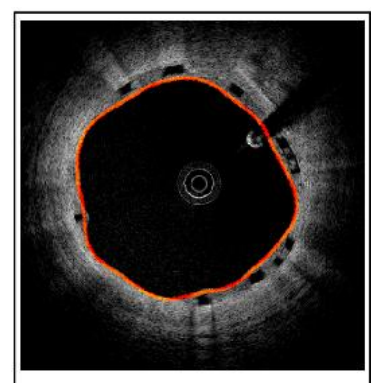

A. Lumen contour at follow-up
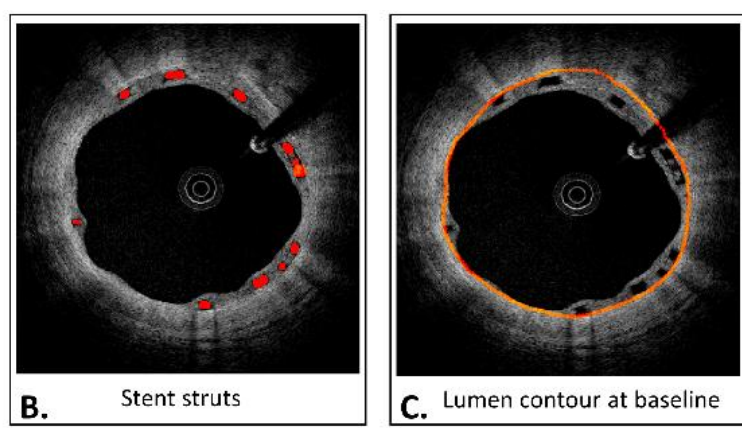

C. Lumen contour at baseline

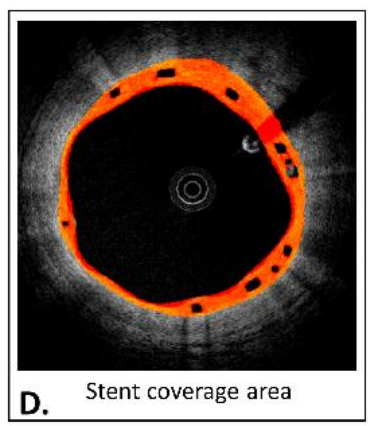

Figure 3. Definition of lumen contour at 9-months follow-up (A), stent struts (B), and at baseline (C). The restenosis area (D) was obtained from the pixels between contours that were subtracted with the stent struts.

\section{Results}

Blood velocity patterns and TAWSS distribution at baseline are shown in Figures 4 and 5 . Blood flow into the diagonal branch was disrupted by the presence of the scaffold over the orifice, leading to micro-recirculatory zones. The $55.6 \%$ of the scaffolded segment was exposed to TAWSS lower than $0.4 \mathrm{~Pa}$. The distribution of TAWSS at the scaffolded main branch was reported in terms of percentage area (Figure 6).

Imaging after 9 months showed a luminal narrowing of 19\% in the LAD and $0 \%$ across the ostium of the diagonal by QCA. Strut coverage was assessed by measuring NIT on top of the struts on the OCT images, reported in Figure 6. At follow-up, there was a good healing response with complete coverage in $99 \%$ of struts. The distal segment had a wider distribution of neointimal growth and this was associated with a higher baseline TAWSS. The percentage restenosis area is reported in Figure 7, with the entire scaffolded segment and at each region.

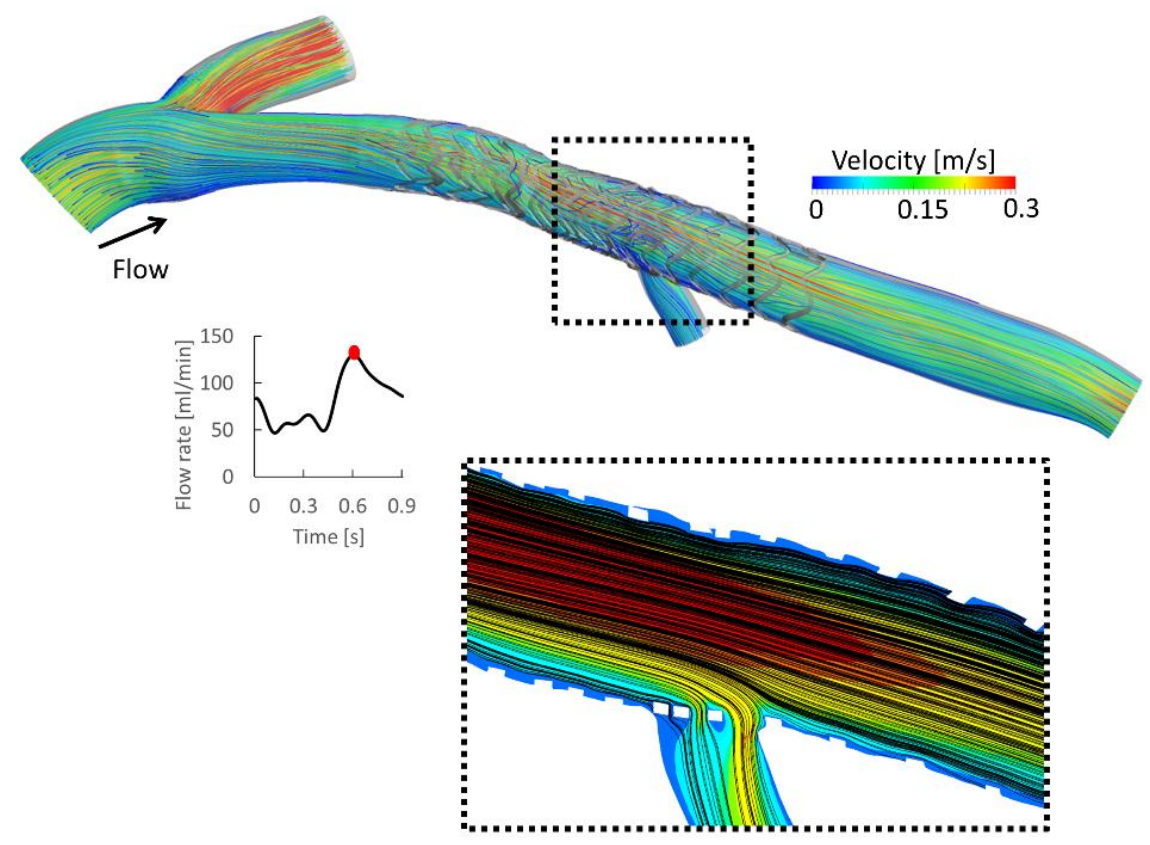

Figure 4. Streamlines of the velocity colored by the velocity magnitude (top) and the in-plane velocity contour map with in-plane streamlines (bottom) at peak flow-rate. 


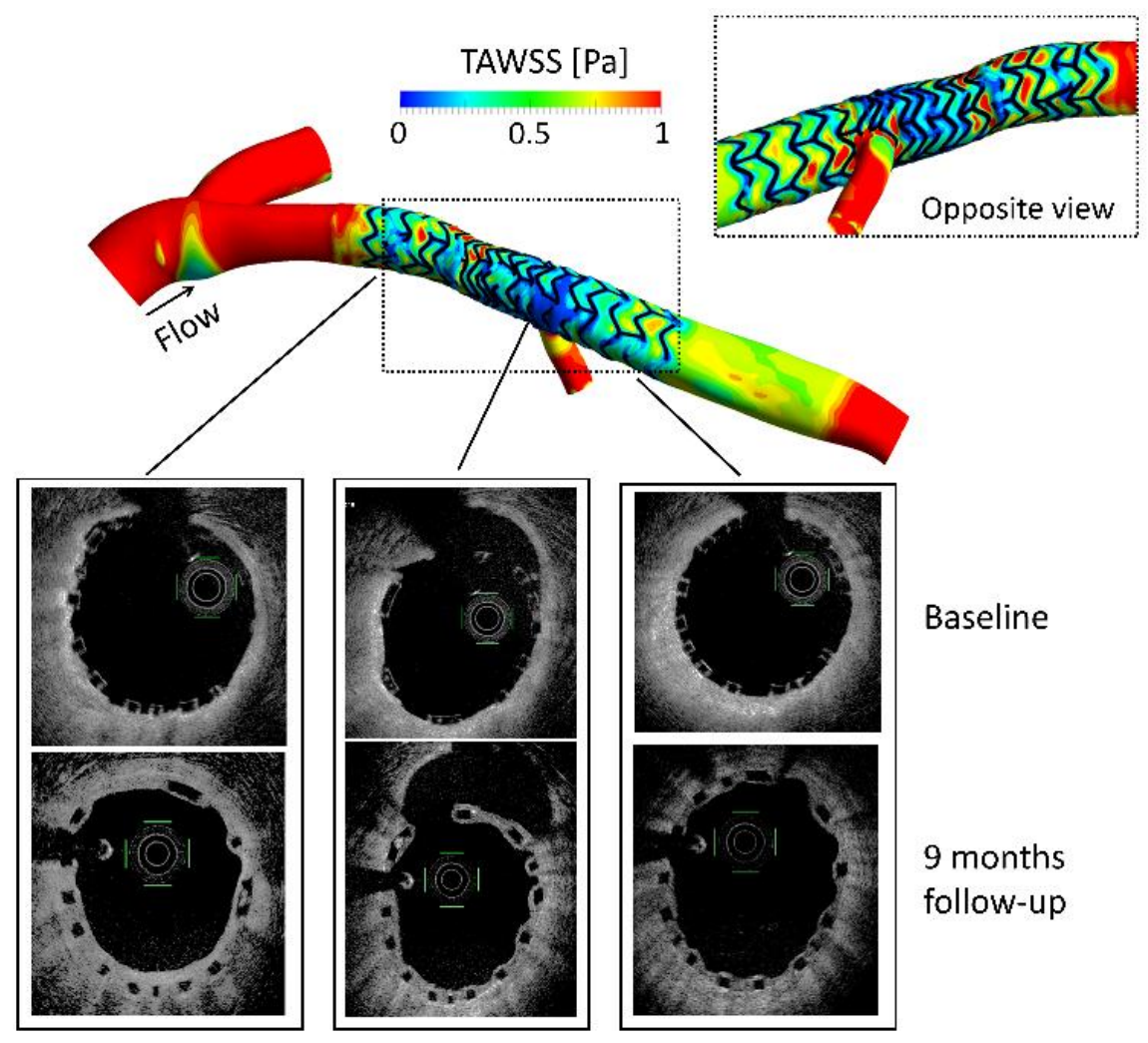

Figure 5. Contour map of the time-averaged wall shear stress (TAWSS) with an enlargement of the opposite view of the bifurcation (top). Baseline and follow-up OCT images at the proximal, bifurcation, and distal regions, respectively.
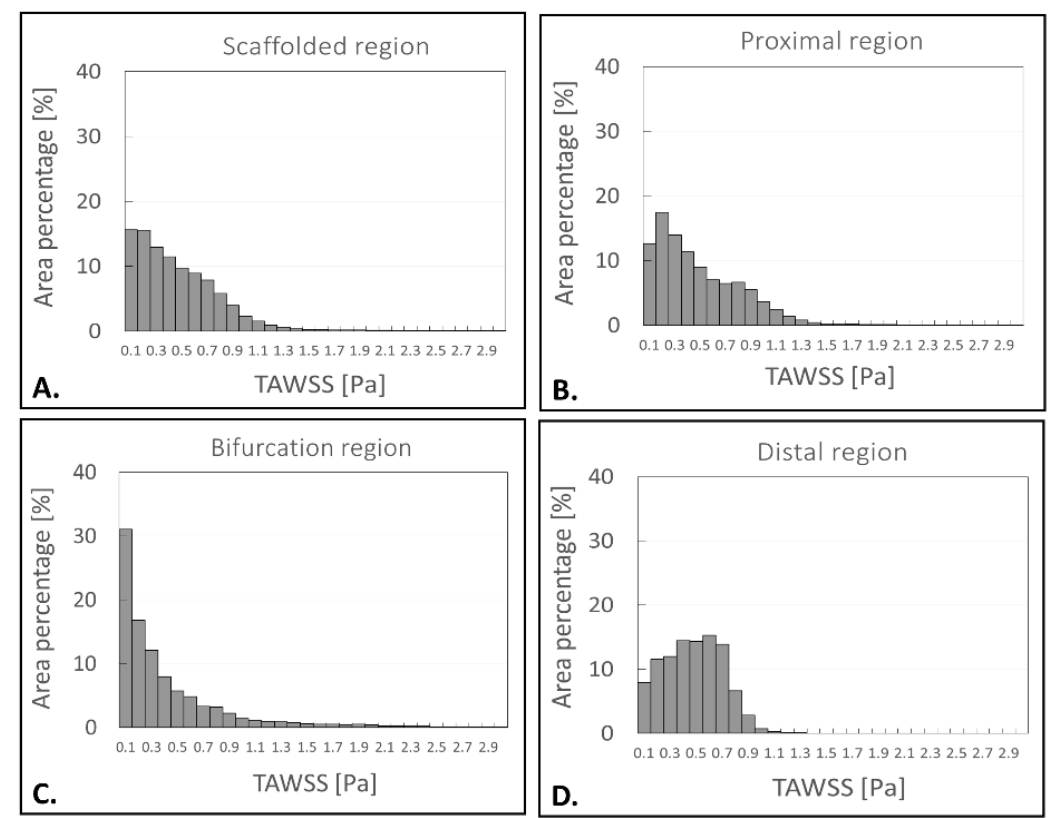

Figure 6. Histograms of the scaffolded surface percentage area under intervals of time-averaged wall shear stress (TAWSS) from $0 \mathrm{~Pa}$ and $3 \mathrm{~Pa}$. (A): Scaffolded region of the main branch; (B): Proximal region of the scaffolded main branch; $(\mathbf{C})$ : Bifurcation region of the scaffolded main branch; (D): Distal region of the scaffolded main branch. 

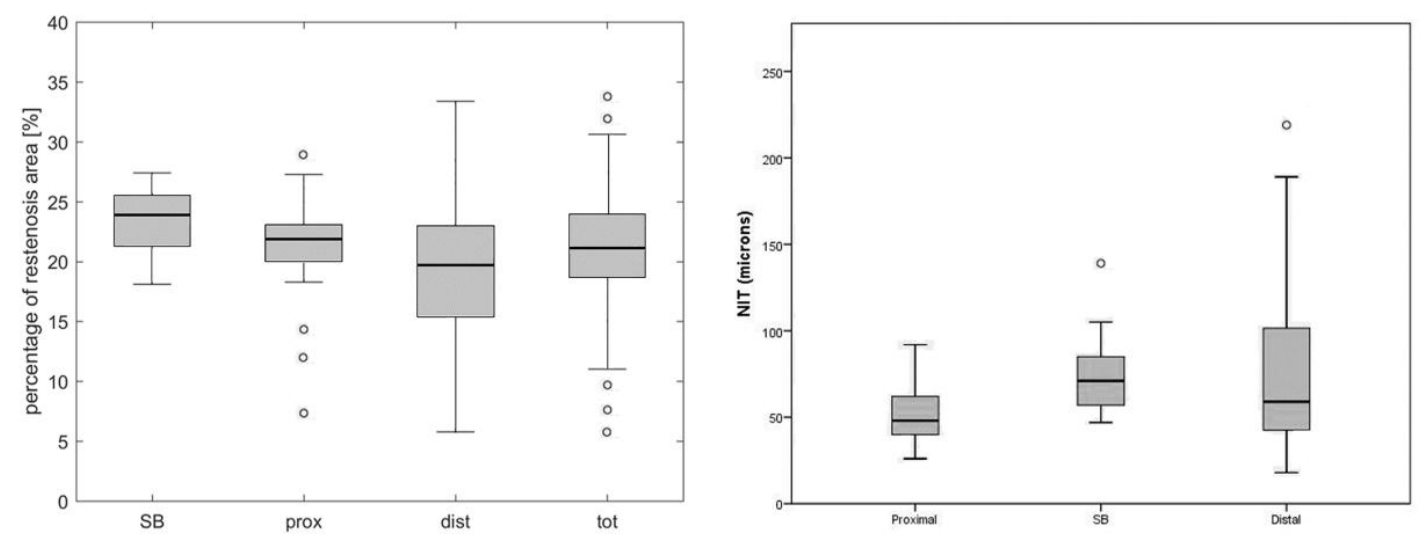

Figure 7. Boxplots of the computed percentage of restenosis area (left) and neo-intimal thickening (right) grouped according to the position. Results were grouped in side branch (SB), scaffolded proximal (prox) and distal (dist) regions, as well as the percentage of restenosis area was reported also for the entire scaffolded region (tot).

\section{Discussion}

In this study, a validated OCT-based reconstruction methodology was employed to analyze a scaffolded coronary artery geometry involving a bifurcation. The actual configuration of the implanted Absorb BVS was part of the physical domain and patient's hemodynamics was simulated with a commercial CFD software. Then, it was demonstrated: (i) The possibility of calculating the WSS distribution and blood flow patterns in bifurcation segments treated with an Absorb BVS; (ii) the potential application of such simulations in the clinical setting by matching the numerical methods with imaging and clinical outcomes in patients. In the authors' opinions, the proposed approach can improve the understanding of the hemodynamic impact of any scaffold design at coronary bifurcations. The additional 9-months follow-up intravascular images enabled the quantification of vessel remodeling after the implantation of a bioresorbable scaffold when treating non-complex bifurcations with a provisional strategy.

In the literature, the effect of the presence of Absorb BVS on WSS has been limited to relatively straight coronary vessels without bifurcations and the CFD physical domain did not include the scaffold, as the innermost lumen boundary was traced. Karanasos and colleagues [18] evaluated the effect of WSS after two years from the intervention in the scaffolded segment. Results were compared with the vascular healing response of eight patients from the ABSORB A cohort that underwent 5-year invasive follow-up OCT acquisition. It was found that regions exposed to low shear stress had thinner fibrous caps. In 12 patients from the ABSORB B cohort, Bourantas and colleagues [17] investigated the effect of WSS after BVS implantation on neointimal growth at 1 year. Similar to studies with metal stents, areas that were exposed to low WSS showed more intimal thickening at follow-up. Analysis of the peri-strut flow distribution showed regions of low WSS as well as recirculation zones on both sides of the struts, and regions of high WSS at the top. This hemodynamic profile may explain the higher thrombotic rate of the Absorb BVS compared to drug eluting stents [19]. One of the postulated mechanisms is the activation of platelets induced by high WSS on top of struts and sequestration in low recirculation zones adjacent to struts causing thrombus formation [20].

According to the most recent computational works about the application of polymeric bioresorbable scaffolds, such as the work of Thondapu and co-authors [21], and the review presented by Chiastra and colleagues [3], the study here described is the first that includes the reconstruction of coronary bifurcations with an implanted scaffold, the actual deployed configuration of which was included in the model [6]. Nevertheless, the applied method has limitations related to the periodic deformation of the coronary artery that affects the change of lumen cross-section area during the OCT pullback. Moreover, rigid wall conditions, with the coronary artery model fixed in the space domain, 
were assumed for the CFD simulation. Although it has been shown that the assumption of rigid walls is acceptable in case of stented geometries [21], it is still unclear whether the coronary motion due to heart contraction can be neglected [3]. The performed CFD simulation resembled the flow condition at baseline. The scaffold bioresorption process was not modeled. Finally, it was not possible to associate each baseline and follow-up OCT images to compute the neo-intima thickening. Hence, we defined the baseline lumen contour from the follow-up OCT image dataset, as done in a previous study [17].

\section{Conclusions}

The present study illustrated the use of a patient-specific technique to model blood flow in a scaffolded coronary bifurcation segment. As this is a case report, results from the comparison between TAWSS and NIT could not be generalized to larger number of patients. However, the described approach provided valuable complementary information, which might improve the clinical outcomes in this subset of coronary artery diseases.

Author Contributions: Conceptualization, S.M., R.R., F.B., F.M. and C.C.; Methodology, S.M., M.B., E.M., C.C.; Software, M.B., S.M., E.M., C.C.; Validation, R.R., S.M. and C.C.; Formal Analysis, S.M., R.R.; Writing-Original Draft Preparation, S.S., R.R. and C.C.; Writing-Review \& Editing, C.C., G.D., F.M., L.M., D.H.-S., F.B., J.C.; Funding Acquisition, G.D.

Funding: S.M. is supported by the European Commission through the H2020 Marie Skłodowska-Curie European Training Network H2020-MSCA-ITN-2014 VPH-CaSE, www.vph-case.eu, GA No. 642612.

Conflicts of Interest: The authors declare no conflict of interest.

\section{References}

1. Sotomi, Y.; Onuma, Y.; Collet, C.; Tenekecioglu, E.; Virmani, R.; Kleiman, N.S.; Serruys, P.W. Bioresorbable Scaffold: The Emerging Reality and Future Directions. Circ. Res. 2017, 120, 1341-1352. [CrossRef] [PubMed]

2. Van der Heiden, K.; Gijsen, F.J.H.; Narracott, A.; Hsiao, S.; Halliday, I.; Gunn, J.; Wentzel, J.J.; Evans, P.C. The effects of stenting on shear stress: Relevance to endothelial injury and repair. Cardiovasc. Res. 2013, 99, 269-275. [CrossRef] [PubMed]

3. Chiastra, C.; Migliori, S.; Burzotta, F.; Dubini, G.; Migliavacca, F. Patient-Specific Modeling of Stented Coronary Arteries Reconstructed from Optical Coherence Tomography: Towards a Widespread Clinical Use of Fluid Dynamics Analyses. J. Cardiovasc. Transl. Res. 2018, 11, 156-172. [CrossRef] [PubMed]

4. Nammas, W.; Ligthart, J.M.R.; Karanasos, A.; Witberg, K.T.; Regar, E. Optical coherence tomography for evaluation of coronary stents in vivo. Expert Rev. Cardiovasc. Ther. 2013, 11, 577-588. [CrossRef] [PubMed]

5. Malek, A.M.; Alper, S.L.; Izumo, S. Hemodynamic shear stress and its role in atherosclerosis. JAMA 1999, 282, 2035-2042. [CrossRef] [PubMed]

6. Migliori, S.; Chiastra, C.; Bologna, M.; Montin, E.; Dubini, G.; Aurigemma, C.; Fedele, R.; Burzotta, F.; Mainardi, L.; Migliavacca, F. A framework for computational fluid dynamic analyses of patient-specific stented coronary arteries from optical coherence tomography images. Med. Eng. Phys. 2017, 47, 105-116. [CrossRef] [PubMed]

7. Medina, A.; Suárez de Lezo, J.; Pan, M. A new classification of coronary bifurcation lesions. Rev. Esp. Cardiol. 2006, 59, 183. [CrossRef] [PubMed]

8. Wang, A.; Nakatani, S.; Eggermont, J.; Onuma, Y.; Garcia-Garcia, H.M.; Serruys, P.W.; Reiber, J.H.C.; Dijkstra, J. Automatic detection of bioresorbable vascular scaffold struts in intravascular optical coherence tomography pullback runs. Biomed. Opt. Express 2014, 5, 3589. [CrossRef] [PubMed]

9. Chiastra, C.; Montin, E.; Bologna, M.; Migliori, S.; Aurigemma, C.; Burzotta, F.; Celi, S.; Dubini, G.; Migliavacca, F.; Mainardi, L. Reconstruction of stented coronary arteries from optical coherence tomography images: Feasibility, validation, and repeatability of a segmentation method. PLoS ONE 2017, 12, e0177495. [CrossRef] [PubMed]

10. Chiastra, C.; Morlacchi, S.; Pereira, S.; Dubini, G.; Migliavacca, F. Computational fluid dynamics of stented coronary bifurcations studied with a hybrid discretization method. Eur. J. Mech. B/Fluids 2012, 35, 76-84. [CrossRef] 
11. Davies, J.E.; Whinnett, Z.I.; Francis, D.P.; Manisty, C.H.; Aguado-Sierra, J.; Willson, K.; Foale, R.A.; Malik, I.S.; Hughes, A.D.; Parker, K.H.; et al. Evidence of a dominant backward-propagating "suction" wave responsible for diastolic coronary filling in humans, attenuated in left ventricular hypertrophy. Circulation 2006, 113, 1768-1778. [CrossRef] [PubMed]

12. Sakamoto, S.; Takahashi, S.; Coskun, A.U.; Papafaklis, M.I.; Takahashi, A.; Saito, S.; Stone, P.H.; Feldman, C.L. Relation of Distribution of Coronary Blood Flow Volume to Coronary Artery Dominance. Am. J. Cardiol. 2013, 111, 1420-1424. [CrossRef] [PubMed]

13. Van der Giessen, A.G.; Groen, H.C.; Doriot, P.-A.; de Feyter, P.J.; van der Steen, A.F.W.; van de Vosse, F.N.; Wentzel, J.J.; Gijsen, F.J.H. The influence of boundary conditions on wall shear stress distribution in patients specific coronary trees. J. Biomech. 2011, 44, 1089-1095. [CrossRef] [PubMed]

14. Caputo, M.; Chiastra, C.; Cianciolo, C.; Cutrì, E.; Dubini, G.; Gunn, J.; Keller, B.; Migliavacca, F.; Zunino, P. Simulation of oxygen transfer in stented arteries and correlation with in-stent restenosis. Int. J. Numer. Methods Biomed. Eng. 2013, 29, 1373-1387. [CrossRef] [PubMed]

15. Chiastra, C.; Migliavacca, F. Modeling of blood flow in stented coronary arteries. In Heat Transfer and Fluid Flow in Biological Processes; Becker, S.M., Kuznetsov, A.V., Eds.; Elsevier: Amsterdam, The Netherlands, 2015; pp. 335-370, ISBN 9780124080775.

16. Bourantas, C.V.; Papafaklis, M.I.; Kotsia, A.; Farooq, V.; Muramatsu, T.; Gomez-Lara, J.; Zhang, Y.J.; Iqbal, J.; Kalatzis, F.G.; Naka, K.K.; et al. Effect of the endothelial shear stress patterns on neointimal proliferation following drug-eluting bioresorbable vascular scaffold implantation: An optical coherence tomography study. JACC Cardiovasc. Interv. 2014, 7, 315-324. [CrossRef] [PubMed]

17. Karanasos, A.; Schuurbiers, J.C.H.; Garcia-Garcia, H.M.; Simsek, C.; Onuma, Y.; Serruys, P.W.; Zijlstra, F.; Van Geuns, R.-J.; Regar, E.; Wentzel, J.J. Association of wall shear stress with long-term vascular healing response following bioresorbable vascular scaffold implantation. Int. J. Cardiol. 2015, 191, 279-283. [CrossRef] [PubMed]

18. Serruys, P.W.; Chevalier, B.; Sotomi, Y.; Cequier, A.; Carrié, D.; Piek, J.J.; Van Boven, A.J.; Dominici, M.; Dudek, D.; McClean, D.; et al. Comparison of an everolimus-eluting bioresorbable scaffold with an everolimus-eluting metallic stent for the treatment of coronary artery stenosis (ABSORB II): A 3 year, randomised, controlled, single-blind, multicentre clinical trial. Lancet 2016, 388, 2479-2491. [CrossRef]

19. Serruys, P.W.; Suwannasom, P.; Nakatani, S.; Onuma, Y. Snowshoe Versus Ice Skate for Scaffolding of Disrupted Vessel Wall. JACC Cardiovasc. Interv. 2015, 8, 910-913. [CrossRef] [PubMed]

20. Thondapu, V.; Tenekecioglu, E.; Poon, E.K.W.; Collet, C.; Torii, R.; Bourantas, C.V.; Chin, C.; Sotomi, Y.; Jonker, H.; Dijkstra, J.; et al. Endothelial shear stress 5 years after implantation of a coronary bioresorbable scaffold. Eur. Heart J. 2018, 39, 1602-1609. [CrossRef] [PubMed]

21. Chiastra, C.; Migliavacca, F.; Martinez, M.A.; Malve, M. On the necessity of modelling fluid-structure interaction for stented coronary arteries. J. Mech. Behav. Biomed. Mater. 2014, 34, 217-230. [CrossRef] [PubMed]

(C) 2018 by the authors. Licensee MDPI, Basel, Switzerland. This article is an open access article distributed under the terms and conditions of the Creative Commons Attribution (CC BY) license (http://creativecommons.org/licenses/by/4.0/). 